\title{
Withdrawal of maintenance digoxin from institutionalized elderly
}

\author{
Colin Macarthur
}

Department of Epidemiology and Biostatistics, McGill University, Montreal, Canada

\begin{abstract}
Summary: Chronic use of digitalis is common amongst the institutionalized elderly. Associated with digitalis use is the potential for toxicity and/or adverse reactions. In this study maintenance digoxin was withdrawn from 14 elderly nursing home residents in sinus rhythm. Subjects were followed over 18 months for evidence of dysrhythmia or heart failure. One resident had an episode of supraventricular tachycardia which required digoxin to be restarted. One resident developed left heart failure, treated with oral diuretic. For 12 of the 14 residents, withdrawal of maintenance digoxin was achieved without deleterious effect. This study suggests that maintenance digoxin may be withdrawn safely in elderly nursing home residents in sinus rhythm and without history of atrial dysrhythmia.
\end{abstract}

\section{Introduction}

It is more than 200 years since William Withering described the medicinal uses of powdered extract of purple foxglove (Digitalis purpurea) on dropsy and other diseases. ${ }^{1}$ From this time, the use of digitalis has been widespread. In 1982, over all ages, digoxin was the tenth most frequently prescribed medication in the United States, totalling 25.6 million prescriptions. ${ }^{2}$ Chronic digitalis use is particularly common in the elderly. Studies of populations of elderly living in the community, ${ }^{3}$ admitted to geriatric wards, ${ }^{4}$ and institutionalized ${ }^{5}$ have demonstrated digitalis use by $6.9 \%, 15 \%$, and $21.8 \%$ of these elderly populations respectively.

However, the hazards associated with digitalis use are numerous and potentially life-threatening, even with cautious use. Contributing factors include: the narrow therapeutic range, the interindividual variation in tissue sensitivity and the potential for drug interactions. ${ }^{6}$ The potential for digitalis toxicity in the elderly is also compounded by the altered pharmacokinetics of digoxin associated with increasing age. Delayed renal clearance may increase the half-life (and therefore serum level for a given dose) of digoxin by up to $40 \%$ in the elderly. ${ }^{7}$ Studies have indicated adverse drug reactions in up to $29 \%$ of institutionalized elderly patients prescribed maintenance digitalis. ${ }^{8}$

The purpose of this study was to evaluate, over

Correspondence: C. Macarthur, M.B., Ch.B., M.Sc., 751 Rue Agnes, Montreal, Quebec H4C 2P9, Canada Accepted: 7 June 1990
18 months, the effects of withdrawal of maintenance digoxin in elderly nursing home residents in sinus rhythm.

\section{Methods}

Forest Grove Care Centre is a private-for-profit nursing home providing supervised personal care for persons who require assistance in coping with the activities of daily living, but who are not sufficiently ill to warrant general or auxiliary hospital admission. Those residents on maintenance digoxin were identified using the monthly pharmacy record (which details prescription medications for each resident). Informed consent for inclusion in the study was obtained from all such residents and the option to withdraw at any point in the study was made clear. The medical chart for each resident was reviewed and a detailed history and complete physical examination was performed on each subject. Baseline serum digoxin levels for each subject were determined by averaging the results of two venous samples, each taken at approximately 6 hours post-dosage. Additional laboratory tests included: a chest X-ray (CXR) and electrocardiogram (ECG) examined by an independent observer, serum electrolytes, blood urea nitrogen and creatinine. Residents with evidence of atrial fibrillation or atrial dysrhythmia were excluded from the study. 


\section{Results}

From a total of 142 residents, $20(14 \%)$ were noted to be on maintenance digoxin: 19 white females and 1 white male. All residents agreed to participate in the study. Six subjects were excluded from the study: 3 had evidence of atrial fibrillation (two females, one male), 2 subjects died prior to the study beginning and one subject was transferred to auxiliary care and lost to follow up. The 14 study subjects remaining were all female, with an average age of 82.5 years $(67-92)$. Seven of the 14 subjects $(50 \%)$ were over 85 years of age.

No adverse reactions or toxic effects of digoxin were noted in the study population. The original reason for prescription of digoxin was known in 5 $(36 \%)$ of the 14 subjects, with 'heart failure' the sole recorded indication. The chest X-rays showed no evidence of pulmonary congestion whilst the ECGs showed evidence of previous myocardial infarction in 2 patients. The serum digoxin was in the therapeutic range in 12 patients and below it in two. Twelve of the 14 subjects $(86 \%)$ who had their maintenance digoxin withdrawn remained well over the 18 months of follow-up. No clinical or chest X-ray evidence of left heart failure or clinical evidence of dysrhythmias was noted for any subject following withdrawal. Exercise tolerance was also unchanged for the 12 subjects.

One subject was hospitalized with an episode of supraventricular tachycardia (SVT), 4 weeks following withdrawal of digoxin. The episode required intravenous digoxin and verapamil to convert and the patient was maintained on digoxin following the episode. One subject developed clinical evidence of left heart failure 6 weeks following withdrawal of digoxin which responded to $40 \mathrm{mg}$ of oral furosemide daily.

\section{Discussion}

Extensive digitalis use in the elderly is not surprising given the two principal indications for digitalis, namely, the treatment of arrhythmias and the treatment of heart failure. Up to $85 \%$ of patients with heart failure are $\geqslant 65$ years of age, and the prevalence of atrial fibrillation also increases with age. ${ }^{9.10}$ The main anti-arrhythmic use of digitalis is in the management of ventricular response in atrial fibrillation. " The clinical application of digitalis in the treatment of heart failure however, is not agreed upon. Cattell and Gold in 1938 demonstrated the inotropic effects of digitalis on the isolated cat papillary muscle. ${ }^{12} \mathrm{~A}$ favourable haemodynamic and clinical response to chronic digoxin therapy may be possible in selected patients with advanced heart failure (that is, persistently elevated left ventricular filling pressures, low car- diac outputs and peripheral vasoconstriction). ${ }^{13}$ However, the issue is whether the positive inotropic effect of digoxin is clinically meaningful for patients with mild heart failure and in normal sinus rhythm. ${ }^{14}$

Following withdrawal of maintenance digoxin, 12 of the $14(86 \%)$ subjects remained clinically well, with no evidence of left heart failure, dysryhthmia or decreased exercise tolerance over the 18 month period of follow-up. These findings support those of other studies indicating that maintenance digitalis may be withdrawn without deleterious effect in elderly patients in sinus rhythm..$^{15-18}$ In addition, this study population consisted of institutionalized elderly, with 6 of the 12 subjects $(50 \%)$ aged 85 years or older. This elderly sub-group, commonly referred to as the 'old old', is recognized as having the highest risk of both illness and adverse drug reactions. ${ }^{19}$ Also, the period of followup for this elderly population was 18 months - up to 3 times longer than other, similar, short-term studies.

Given the limited role of digitalis in the treatment of mild heart failure, the recurrence of failure in one case allowed an alternative, less toxic medication, a diuretic, to be used. Other (possibly even safer) drug therapies for mild heart failure in elderly patients include angiotensin-converting enzyme (ACE) inhibitors and partial $\beta$-agonists. ${ }^{13}$

One subject required digoxin to be restarted following an episode of supraventricular tachycardia (SVT). On communication with the subject's previous general practitioner, a history of SVT in the past was elicited. Withdrawal of maintenance digitalis in patients with a history of atrial dysrhythmia is not indicated..$^{18}$ This incident highlights the importance of obtaining a complete medical history on elderly persons admitted to institutional care - preferably through written communication with the general practitioner best acquainted with the patient. If not obtained, incomplete medical histories are common and often compounded by the high prevalence of dementia among residents. This, in part, may explain why for 9 of the 14 subjects $(64 \%)$, the original reason for prescription of digoxin was unknown. However, in addition, excessive and/or inappropriate prescribing of digoxin for heart failure in the elderly may have contributed to this finding. As regards local prescribing practices, prescription of digoxin has been shown to be commoner in the United States than in Britain. ${ }^{20}$ However, it is also possible that digoxin was inappropriately prescribed for these elderly residents (possibly for peripheral oedema rather than true heart failure), as none of the subjects showed clinical or chest X-ray evidence of left heart failure prior to the study beginning. In this context, prescribing practices in long-term care facilities have been questioned. ${ }^{21}$ 


\section{References}

1. Withering, W. An Account of the Foxglove and Some of its Medical Uses - Practical Remarks on Dropsy and Other Diseases. M. Swinney, Birmingham, 1785.

2. Baum, C., Kennedy, D.L., Forbes, M.B. \& Jones, J.K. Drug use and expenditures in 1982. JAMA 1985, 253: 382-386.

3. Schocken, D.D., Robinson, B.E., Krug-Fite, J., Lyman, G.H. \& Barry, P.P. Digitalis use in a retirement community. $\mathrm{J} \mathrm{Am}$ Geriatr Soc 1986, 34: 504-506.

4. Alexander, N., Goodwin, J.S. \& Currie, C. Comparison of admission and discharge medications in two geriatric populations. J Am Geriatr Soc 1985, 33: 827-832.

5. Fonrose, H.A., Ahlbaum, N., Bugatch, E., Cohen, M., Genovese, C. \& Kelly, J. The efficacy of digitalis withdrawal in an institutionalised population. J Am Geriatr Soc 1974, 22: 208-211.

6. Chamberlain, D.A. Digitalis: Where are we now? (Editorial). Br Heart J 1985, 54: 227-233.

7. Cusack, B., Horgan, J., Kelly, J.G. et al. Pharmacokinetics of Br J Clin Pharmacol 1978, 6: 439-440.

8. Carter, B.L., Small, R.E. \& Garnett, W.R. Monitoring digoxin therapy in two long term care facilities. $J \mathbf{A m}$ Geriatr Soc 1981, 29: 263-268.

9. McKee, P.S., Castelli, W.P., McNamara, P.M. \& Kannel, W.B. The natural history of congestive heart failure: the Framingham Study. $N$ Engl J Med 1971, 285: 1441-1446.

10. Morris, D.C. \& Hurst, J.W. Atrial fibrillation. Curr Probl Cardiol 1980, 5: 5-13.

11. Levitt, B. \& Keefe, D.L. Clinical use of digitalis materials. J Clin Pharmacol 1985, 25: 507-513.
12. Cattell, M. \& Gold, H. The influence of digitalis glycosides on the force of contraction of mammalian cardiac muscle. $J$ Pharmacol Exp Ther 1938, 62: 116-125.

13. Francis, G.S. Which drug for what patient with heart failure and when? Cardiology 1989, 76: 374-383.

14. Hamer, $J$. The paradox of the lack of efficacy of digitalis in congestive heart failure with sinus rhythm. Br J Clin Pharmacol 1979, 8: 109-113.

15. Gheorghiade, M. \& Beller, G.A. Effects of discontinuing maintenance digoxin therapy in patients with ischemic heart disease and congestive heart failure in sinus rhythm. Am J Cardiol 1983, 51: 1243-1250.

16. Johnston, G.D. \& McDevitt, D.G. Is maintenance digoxin necessary in patients with sinus rhythm? Lancet 1979, i $567-570$

17. Boman, K., Allgulander, S. \& Skoglund, M. Is maintenance digoxin necessary in geriatric patients? Acta Med Scand 1981, 210: $493-495$.

18. Taggart, A.J., Johnston, G.D. \& McDevitt, D.G. Digoxin withdrawal after cardiac failure in patients with sinus rhythm. J Cardiovasc Pharmacol 1983, 5: 229-234.

19. Lamy, P.P. The elderly and drug interactions. J Am Geriatr Soc 1986, 34: 586-592.

20. Lawson, D.H. \& Jick, H. Drug prescribing in hospitals: An international comparison. Am J Public Health 1976, 66: 644-648

21. Riesenberg, D. Drugs in the institutionalised elderly - Time to get it right? (Editorial). JAMA 1988, 260: 3054. 